\title{
Epigallocatechin-3-gallate inhibits the invasion of salivary adenoid cystic carcinoma cells by reversing the hypermethylation status of the RECK gene
}

\author{
XIAO-QING ZHOU ${ }^{1 *}$, XIAO-NAN XU $^{1 *}$, LEI LI $^{1}$, JIAN-JUN MA ${ }^{1}$, EN-MING ZHEN ${ }^{1}$ and CHENG-BING HAN ${ }^{2}$ \\ ${ }^{1}$ Department of Oral and Maxillofacial Surgery, Shandong Jining No. 1 People's Hospital, Jining, Shandong 272111; \\ ${ }^{2}$ Department of Oral and Maxillofacial Surgery, Weifang People's Hospital, Weifang, Shandong 261041, P.R. China
}

Received October 24, 2014; Accepted July 23, 2015

DOI: $10.3892 / \mathrm{mmr} .2015 .4213$

\begin{abstract}
Epigallocatechin-3-gallate (EGCG) is an active and major constituent of green tea. As a non-nucleoside inhibitor of DNA methylation, EGCG is able to inhibit the hypermethylation of newly synthesised DNA, resulting in the reversal of hypermethylation and recovery in expression of the silenced genes. Reversion-inducing cysteine-rich protein with Kazal motifs (RECK) is a novel tumour suppressor gene, which negatively regulates matrix metalloproteinases, and inhibits tumour invasion, angiogenesis and metastasis. The present study aimed to examine the effects of EGCG on the methylation status of the RECK gene and tumour invasion in a salivary adenoid cystic carcinoma (SACC) cell line in vitro. Marked levels of methylated and weak levels of unmethylated RECK promoter were detected in the SACC 83 cells, which was determined using methylation-specific polymerase chain reaction (PCR). In addition, the treatment of SACC 83 cells with EGCG partially reversed the hypermethylation status of the RECK gene. Western blot analysis and reverse transcription-PCR demonstrated that EGCG significantly enhanced the protein and mRNA expression levels of RECK, and significantly reduced the invasive ability of the SACC83 cells, as determined using a Transwell assay. These results suggested that EGCG possesses novel anti-metastatic therapeutic potential for the treatment of SACC.
\end{abstract}

Correspondence to: Dr Cheng-Bing Han, Department of Oral and Maxillofacial Surgery, Weifang People's Hospital, 151 Guanwen Road, Weifang, Shandong 261041, P.R. China E-mail: cbhan3270203@163.com

${ }^{*}$ Contributed equally

Key words: RECK, hypermethylation, salivary adenoid cystic carcinoma, epigallocatechin-3-gallate, Transwell assay

\section{Introduction}

Salivary adenoid cystic carcinoma (SACC) is one of the most common types of malignancy, which is known to be associated with persistent slow growth, perineural invasion, high rates of recurrence and the formation of distant metastases (1). Despite the use of aggressive surgery, the 5-year survival rate of SACC is $\sim 75 \%$, whereas the long-term survival rate is only $39.6 \%$ (2). The underlying molecular mechanisms of SACC carcinogenesis remain to be fully elucidated.

Changes in DNA methylation is commonly observed in human cancer $(3,4)$. The hypomethylation of oncogenes can result in their aberrant activation, and hypermethylation of tumour suppressor genes can result in their silencing (5). DNA methylation inhibitors can be divided into two categories: Nucleoside inhibitors and non-nucleoside inhibitors. Nucleoside inhibitors, including 5-aza-2'-deoxycytidine, are associated with substantial toxic effects and have short half-lives in aqueous solutions (6). Epigallocatechin-3-gallate (EGCG) is a non-nucleoside inhibitor (7), which mediates the inhibition of DNA methylation by binding to the catalytic pocket of human DNA methyltransferase (DNMT) and inhibit the hypermethylation of newly synthesised DNA, resulting in the reversal of hypermethylation and the re-expression of silenced genes.

Several oncogene and tumour suppressor gene candidates have been suggested to be involved in SACC, including suprabasin (8), aquaporin 1 (9), phosphatase and tensin homolog deleted on chromosome 10 (10), cyclin-dependent kinase inhibitors (11) and RAS-associated domain family protein 1A (12). The reversion-inducing cysteine-rich protein with Kazal motifs (RECK) gene is anchored to the cell surface by glycosylphosphatidylinositol, which can suppress tumour invasion and metastasis via degradation of the extracellular matrix (13). RECK is expressed in various types of normal human tissue, and is downregulated in several types of human cancer, including pancreatic cancer (14), colorectal cancer (15), breast cancer (16) and hepatocellular carcinoma (17). Our previous study demonstrated that the expression of RECK was significantly lower in SACC, compared with normal tissue (18); however, the underlying mechanism remains to be fully elucidated.

The present study aimed to determine the methylation status of the RECK promoter in the human SACC83 SACC 
cell line, and aimed to investigate the effects of EGCG on the expression of RECK and the invasiveness of the SACC83 cells.

\section{Materials and methods}

Cell lines and cell culture. The SACC83 SACC cell line [kindly provided by Professor Shenglin Li (Department of Oral and Maxillofacial Surgery, School of Stomatology, Beijing University, Beijing, China)] was cultured in Dulbecco's modified Eagle's medium (DMEM) supplemented with 10\% fetal bovine serum (FBS; GE Healthcare Life Sciences, Logan, UT, USA), 50,000 units penicillin and $50 \mathrm{mg}$ streptomycin (both from Sigma-Aldrich, St. Louis, MO, USA) at $37^{\circ} \mathrm{C}$ in a humidified incubator containing $5 \% \mathrm{CO}_{2}$.

EGCG treatment. To determine the dose- and time-dependent changes associated with EGCG treatment, the SACC83 cells were treated in triplicate with EGCG (Wako Pure Chemical Industries, Ltd., Osaka, Japan), as described previously (19). Briefly, the cells were seeded at low density $\left(5 \times 10^{5} / 100 \mathrm{~mm}\right.$ dish) $24 \mathrm{~h}$ prior to treatment with EGCG. To determine the dose-dependent changes, the cells were treated with $0,5,15$ or $45 \mu \mathrm{m}$ EGCG for 6 days. EGCG was added, in new culture medium, to the cells on days 1, 3 and 5. For the assessment of time-dependent changes, the cells were treated with $45 \mu \mathrm{m}$ EGCG for $0,36,72$ or $144 \mathrm{~h}$.

Bisulphite modification and methylation-specific polymerase chain reaction $(P C R ; M S P)$. Genomic DNA was isolated and modified using a $\mathrm{CpGenome} \mathrm{T}^{\mathrm{TM}}$ Direct PrepBisulfite Modification kit (EMD Millipore, Billerica, MA, USA), according to the manufacturers' instructions. The DNA concentration was determined using spectrophotometry. For MSP, the following primer sets (Takara Bio Inc., Dalian, China) were used for methylated DNA: M_RECK, forward 5'-ATAAAGAGTTTTGGTACG GGGTAC-3' and reverse 5'-AAAACCGCGAAATACTCG AA-3'); and the primer sets for unmethylated DNA were as follows: U_RECK, forward 5'-TAAAGAGTTTTGGTATGG GGTATGT-3' and reverse 5'-CTCCAAACCACAAAATAC TCAAA-3'. The MSP reactions were performed in a mixture of $12.5 \mu 12 \mathrm{X} \mathrm{Taq}$ PCR Master mix (Tiangen Biotech, Co., Ltd., Beijing, China) reverse primers $(1 \mu \mathrm{l})$, DNA $(<1 \mu \mathrm{g})$ and distilled water in $25-\mu \mathrm{l}$ volumes in a S1000 (Bio-Rad Laboratories, Inc., Hercules, CA. USA) under the following conditions: $94^{\circ} \mathrm{C}$ for $3 \mathrm{~min}$; followed by $30 \mathrm{cycles}$ at $94^{\circ} \mathrm{C}$ for $30 \mathrm{sec}, 55^{\circ} \mathrm{C}$ for $30 \mathrm{sec}$ and $72^{\circ} \mathrm{C}$ for $60 \mathrm{sec}$; and finally $5 \mathrm{~min}$ at $72^{\circ} \mathrm{C}$. The PCR product lengths for the methylated and unmethylated RECK were 195 and $199 \mathrm{bp}$, respectively. Universal Methylated DNA (EMD Millipore) and normal human blood DNA were used as positive controls for methylated and unmethylated statuses, respectively. The blood was donated by the authors of the present study, and ethical approval was obtained from the ethics committee of Weifang People's Hospital (Weifang, China). A water blank was used as a negative control. The positive and negative controls were used appropriately in each round of MSP. All assays were performed in triplicate. The PCR products were separated on 2\% agarose gels (Zeta Biotechnology, Co., Ltd., Guangzhou, China) and visualised using ethidium bromide (EtBr; Haoran Biological Technology Co., Ltd., Shanghai, China) staining.
$R N A$ extraction and reverse transcription (RT)-PCR. Total RNA was extracted using a Total RNA Extraction kit (Takara Biotechnology Co., Ltd., Dalian, China), according to the manufacturer's instructions. The quantity and quality of the RNA samples were measured using a spectrophotometer and electrophoresis. RECK cDNA was synthesised from $1 \mu \mathrm{g}$ of total RNA using a PrimeScript ${ }^{\mathrm{TM}}$ RT Reagent kit with gDNA Eraser (Takara Biotechnology Co., Ltd.). The following primers (Takara Bio Inc.) were used for PCR: RECK, forward 5'-CCTCAGTGAGCACAGTTCAGA-3' and reverse 5'-GCA GCACACACACTGCTGTA-3'. Reactions were performed in $20 \mu \mathrm{l}$ volumes containing $2 \mathrm{X}$ Mater Mix $(10 \mu \mathrm{l})$, forward and reverse primers $(1 \mu \mathrm{l}), \mathrm{cDNA}(2 \mu \mathrm{l})$ and distilled water under the following conditions: $95^{\circ} \mathrm{C}$ for $10 \mathrm{~min}$; followed by 35 cycles at $95^{\circ} \mathrm{C}$ for $30 \mathrm{sec}, 60^{\circ} \mathrm{C}$ for $30 \mathrm{sec}$ and $72^{\circ} \mathrm{C}$ for $30 \mathrm{sec}$; and finally $7 \mathrm{~min}$ at $72^{\circ} \mathrm{C}$. GAPDH was used as an internal control to estimate the efficiency of cDNA synthesis using the primer: GAPDH, forward 5'-GCAGCACACACACTGCTGTA-3' and reverse 5'-TGTGGTCATGAGTCCTTCCA-3'. The predicted size for the PCR products of RECK and GAPDH were 477 and $512 \mathrm{bp}$, respectively. The PCR products were separated on $2 \%$ agarose gel, stained with $\mathrm{EtBr}$ and visualised under ultraviolet light and images were captured using a Bio-Rad VersaDoc 3000 Imaging system (Bio-Rad Laboratories, Inc.). Image J software (version 1.48u; National Institutes of Health, Bethesda, MD, USA) was used for grey value analysis. The relative mRNA expression levels of RECK were normalised against GAPDH.

Western blot analysis. Following treatment with EGCG, the SACC 83 cells were washed twice with cold phosphate-buffered saline (PBS) and treated with extraction buffer [50 M Tris-HCl (pH 7.4), $150 \mathrm{M} \mathrm{NaCl}, 2 \mathrm{M}$ EDTA and $1 \% \mathrm{NP}-40]$. The cell extractions were subsequently centrifuged at $12,000 \mathrm{x}$ g for $15 \mathrm{~min}$ at $4^{\circ} \mathrm{C}$, and the supernatants were collected. The protein concentration was quantified using a Bicinchoninic Acid Protein Measurement kit (Shenneng Bocai Biology, Co., Ltd., Shanghai, China). Equal quantities (40 $\mu \mathrm{g}$ ) of cellular proteins were subjected to $8 \%$ SDS-PAGE, and transferred onto polyvinylidene fluoride membranes (Santa Cruz Biotechnology, Inc., Dallas, TX, USA). The membranes were blocked with 5\% (w/v) non-fat milk in PBS containing 0.1\% Tween-20 (Shenneng Bocai Biology, Co., Ltd., Shanghai, China) and then incubated with primary antibodies (mouse anti-human RECK polyclonal, cat. no. ab88249; anti-GAPDH, cat. no. sc-365062) at 1:1,000 and room temperature for $1 \mathrm{~h}$. Subsequently, the membranes were incubated with an appropriate horseradish peroxidase-conjugated goat anti-mouse immunoglobulin $\mathrm{G}$ secondary antibody (cat. no. sc-2005) at room temperature for $1 \mathrm{~h}$. The immuno-detected proteins were visualised using enhanced chemiluminescence. Image $\mathbf{J}$ software was used for grey value analysis. The anti-RECK antibody was purchased from Abcam (Cambridge, MA, USA) and the anti-GAPDH and secondary antibodies were obtained from Santa Cruz Biotechnology, Inc.

In vitro invasion assay. To assess the invasive ability of the cells treated with EGCG, Matrigel invasion assays were performed using 8-mm pore filter inserts in 24-well plates (Sigma-Aldrich) coated with Matrigel (BD Biosciences, Franklin Lakes, NJ, USA). The cells were incubated with EGCG $(0,5,15$ or $45 \mu \mathrm{m})$ 


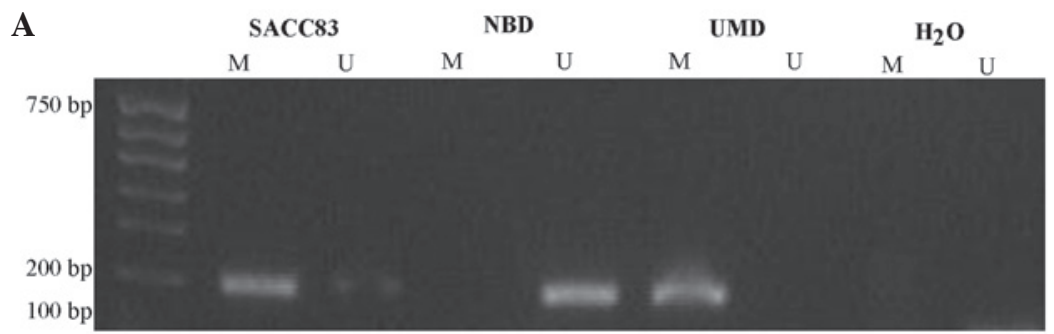

B

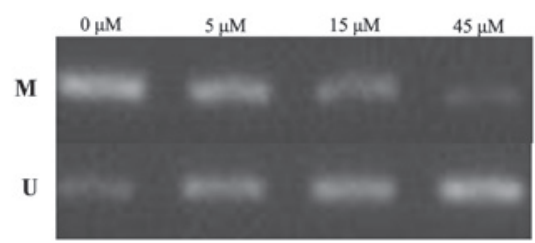

(ACC83) $144 \mathrm{~h}$



(ACC83) $45 \mu \mathrm{M}$ EGCG

Figure 1. Detection of the methylation status of the RECK gene in SACC83 cells. (A) Methylation status of the RECK gene in SACC83 cells, NBD positive control for unmethylatiom, UMN positive control for methylation and $\mathrm{H}_{2} \mathrm{O}$ negative control groups. (B) Changes in the methylation status of the RECK gene in SACC83 cells following treatment with 0,5, 15 or $45 \mu \mathrm{m}$ EGCG for $144 \mathrm{~h}$, or treatment with $45 \mu \mathrm{m}$ EGCG for 0, 36,72 or $144 \mathrm{~h}$. RECK, reversion-inducing cysteine-rich protein with Kazal motifs; EGCG, epigallocatechin-3-gallate; SACC, salivary adenoid cystic carcinoma; M, methylation-specific band; U, unmethylation-specific band; NBD, normal blood DNA; UMD, Universal Methylated DNA; Bp, base pairs.

for $72 \mathrm{~h}$, and were subsequently collected, washed three times with PBS and re-suspended in serum-free DMEM. A total of $1 \times 10^{5}$ cells $/ 200 \mu \mathrm{l}$ medium were plated in the upper chamber of the Transwell unit and allowed to invade for $24 \mathrm{~h}$ at $37^{\circ} \mathrm{C}$. The lower chamber of the Transwell unit was filled with $500 \mu \mathrm{l}$ medium supplemented with $10 \%$ FBS. At the end of the incubation period, the non-invaded cells on the upper surface of the membrane were carefully removed using a cotton swab. The invaded cells on the bottom surface of the membrane were fixed in $4 \%$ formaldehyde for $20 \mathrm{~min}$ and stained with $0.1 \%$ crystal violet for $5 \mathrm{~min}$. Subsequently, the invaded cells on the lower surface of the membrane were visualised in five randomly-selected fields under a microscope (Olympus BH2; Olympus, Tokyo, Japan; magnification, x200). All assays were performed in triplicate and the mean number of invaded cells was used for analysis.

Statistical analysis. Statistical analysis was performed using SPSS 13.0 for Windows (SPSS, Inc., Chicago, IL, USA). Data are expressed as the mean \pm standard deviation. Differences between the groups were assessed using one-way analysis of variance with Dunnett's post-hoc test. $\mathrm{P}<0.05$ was considered to indicate a statistically significant difference.

\section{Results}

Methylation status of the RECK gene in SACC83 cells. The RECK gene in SACC83 cells exhibited marked expression of methylated and weak expression of unmethylated promoter, determined using MSP (Fig. 1A). Normal human blood DNA was used as a positive control for unmethylation, and Universal Methylated DNA was used as a positive control for methylation. $\mathrm{H}_{2} \mathrm{O}$ was used as a negative control.

EGCG reverses the hypermethylation status of $R E C K$ in the SACC83 cell line. The present study aimed to examine the time-and dose-dependent effects of EGCG in SACC83 cells.
A

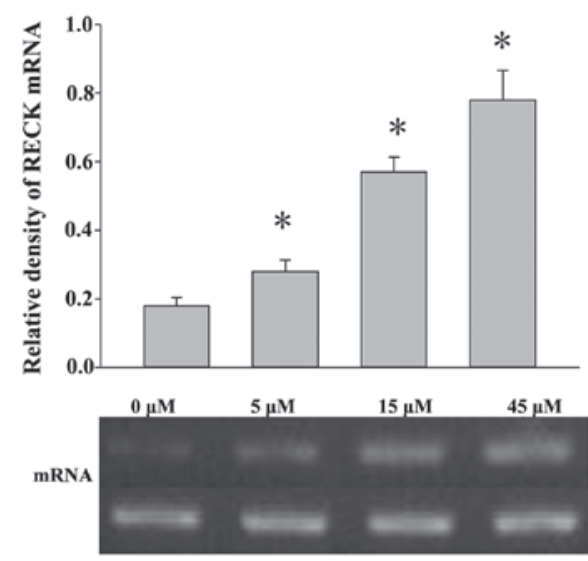

B
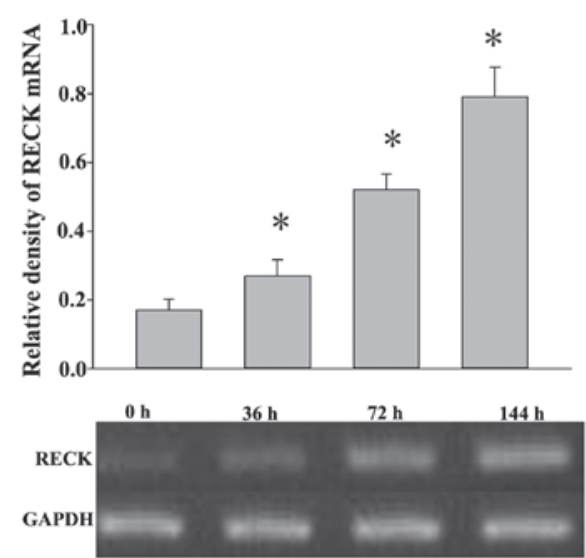

Figure 2. Relative mRNA expression levels of RECK, determined using reverse transcription-quantitative polymerase chain reaction in SACC83 cells. Treatment with EGCG enhanced the mRNA expression of RECK in a (A) dose- and (B) time-dependent manner. The relative mRNA expression levels of RECK were normalised to GAPDH. Band density was determined using densitometry. Data are presented as the mean \pm standard deviation $(n=3) .{ }^{*} \mathrm{P}<0.05$, compared with the control. RECK, reversion-inducing cysteine-rich protein with Kazal motifs; EGCG, epigallocatechin-3-gallate; SACC, salivary adenoid cystic carcinoma. 

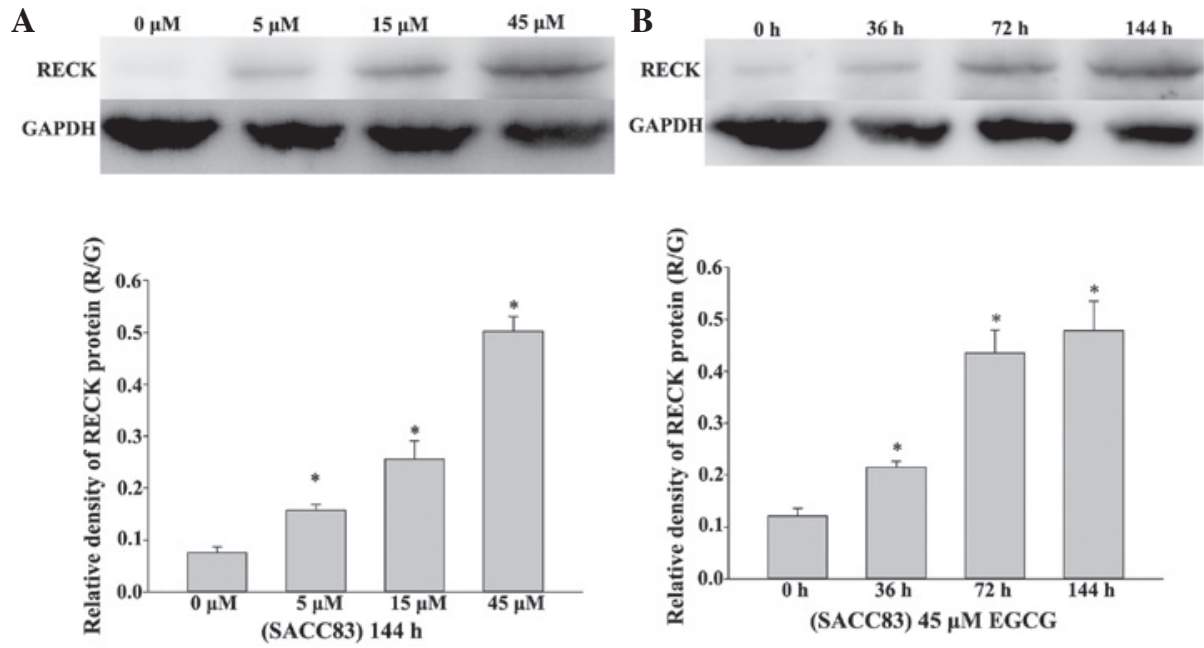

Figure 3. Western blotting was used to detect the protein expression levels of RECK in SACC83 cells following treatment with (A) 0,5, 15 or $45 \mu \mathrm{m}$ EGCG, or treatment with $45 \mu \mathrm{m}$ EGCG for (B) $0,36,72$ or $144 \mathrm{~h}$. R/G indicates the density ratio of the RECK protein (106 kDa), vs. GAPDH (36 kDa). Data are presented as the mean \pm standard deviation. ${ }^{*} \mathrm{P}<0.05$, compared with the control. RECK, reversion-inducing cysteine-rich protein with Kazal motifs; EGCG, epigallocatechin-3-gallate; SACC, salivary adenoid cystic carcinoma.

A
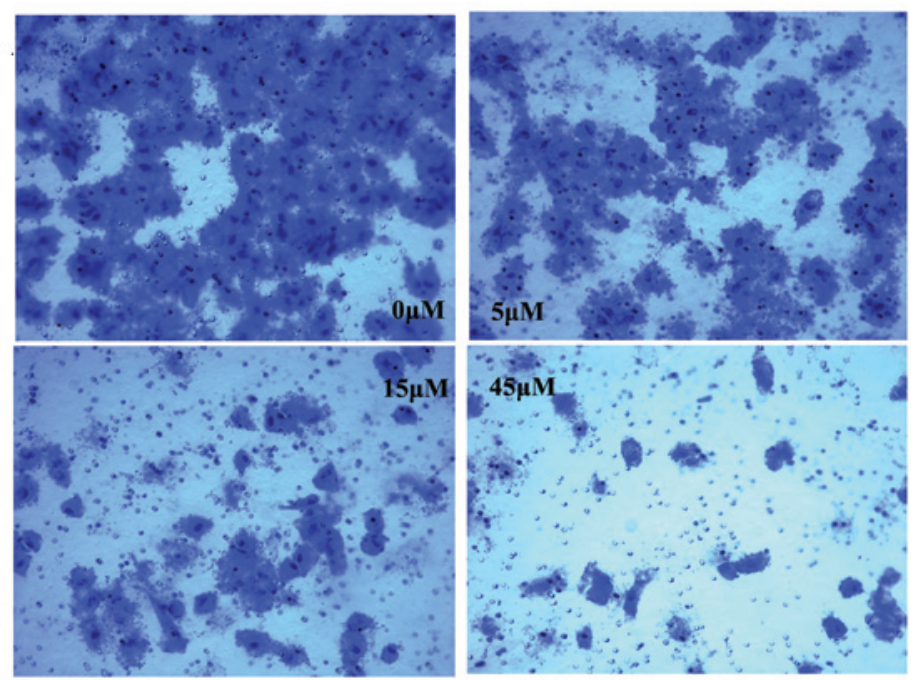

B



Figure 4. EGCG inhibits the invasive ability of SACC83 in a dose-dependent manner. The cells were incubated with different concentrations of EGCG for $72 \mathrm{~h}$. After $24 \mathrm{~h}$, (A) images of the cells were captured and the (B) numbers of invaded cells were counted (magnification, x200). Data are presented as the mean \pm standard deviation. "P $<0.05$, compared with the control. RECK, reversion-inducing cysteine-rich protein with Kazal motifs; EGCG, epigallocatechin-3-gallate; SACC, salivary adenoid cystic carcinoma.

Following treatment of the cells with $0,5,15$ or $45 \mu \mathrm{m}$ EGCG for $144 \mathrm{~h}$, methylation-specific bands of the RECK gene were weak in appearance, whereas unmethylation-specific bands of the RECK gene appeared markedly enhanced. Following treatment of the cells with $45 \mu \mathrm{m}$ EGCG for $0,36,72$ or $144 \mathrm{~h}$, the unmethylation-specific bands of RECK were more marked, whereas the methylation-specific bands of RECK were almost undetectable (Fig. 1B).

Treatment with EGCG enhances the mRNA and protein expression levels of RECK in SACC83 cells. To determine the effects of EGCG on the mRNA expression of RECK, RT-qPCR was 
performed to detect the mRNA expression levels of RECK in the SACC83 cells following treatment with a range of concentrations of EGCG for different durations (Fig. 2). The relative mRNA expression levels of RECK increased in a dose- and time-dependent manner (Fig. 2A and B).

As shown in Fig. 3, the protein expression levels of RECK were relatively low in the untreated SACC83 cells. Following treatment of the cells with different doses of EGCG for 6 days, and with $45 \mu \mathrm{m}$ EGCG for different periods of time, the protein expression levels of RECK increased in the SACC83 cells (Fig. 3A and B).

EGCG reduces the invasiveness of human SACC83 cells. The present study also investigated the effects of EGCG on cell invasion. As shown in Fig. 4, treatment with 5, 15 or $45 \mu \mathrm{m}$ EGCG markedly suppressed the invasive ability of the SACC83 cells in a dose-dependent manner. These results suggested that restoration of the expression of RECK by EGCG is important for inhibiting the invasiveness of SACC83 cells.

\section{Discussion}

RECK is an important matrix metalloproteinase inhibitor, which is involved in the regulation of various physiological and pathological processes. Oh et al (20) previously reported that mice lacking the expression of RECK succumb to mortality in utero due to developmental defects in blood vessels, the neural tube and mesenchymal tissues. In addition, RECK has been identified as a target of myogenic regulatory factors and is involved in the control of myogenesis (21).

Several studies have demonstrated that RECK mRNA and protein are highly expressed in human tissue and untransformed cells (22); however, the expression of RECK is lost or undetected in the majority of tumour cells. Several hypotheses have been suggested regarding the mechanism underlying the low expression levels of RECK in tumour tissues. DNA methylation, which is a critical epigenetic alteration, is associated with the silencing of tumour suppressor genes in several types of cancer. Cho et al (22) demonstrated that downregulation of the mRNA and protein expression levels of RECK in colon tumour tissue are significantly correlated with methylation of the RECK promoter. Furthermore, Chang et al (23) reported that downregulation of the metastasis suppressor RECK is due to promoter methylation in non-small cell lung cancer. In the present study, the methylation status of the RECK promoter was determined using MSP, and the SACC83 cells were found to exhibit weak expression levels of unmethylated promoter and marked expression levels of methylated promoter. These results suggested that a decrease or deficiency in the expression of RECK in SACC83 cells may be caused by the methylation of $\mathrm{CpG}$ islands in the RECK promoter region.

EGCG is a major polyphenol in green tea, and a key active ingredient (19). Previous studies $(24,25)$ have demonstrated that EGCG inhibits DNMT and inhibits the hypermethylation of newly synthesised DNA, resulting in the reversal of hypermethylation and the re-expression of silenced genes, with fewer side effects and toxicity. The present study demonstrated that almost no RECK protein was detected in untreated SACC83 cells. However, following treatment of the cells with EGCG, the mRNA and protein expression levels of RECK increased in a dose- and time-dependent manner in the SACC83 cells. Furthermore, following treatment of SACC83 cells with EGCG, their invasive capability was significantly reduced.

In conclusion, the results of the present study demonstrated that EGCG inhibited cancer cell invasion through reversal of the hypermethylation status of RECK. This may offer a potential therapeutic strategy for the chemotherapeutic treatment of SACC. Further investigations are required to fully elucidate the underlying molecular mechanisms by which EGCG inhibits tumour invasion.

\section{Acknowledgements}

This study was supported by grants from the Science and Technology Development Planning Project of Jining City (no. 2014jnwk03).

\section{References}

1. Gondivkar SM, Gadbail AR, Chole R and Parikh RV. Adenoid cystic carcinoma: A rare clinical entity and literature review. Oral Oncol 47: 231-236, 2011.

2. Fordice J, Kershaw C, El-Naggar A and Goepfert H: Adenoid cystic carcinoma of the head and neck: Predictors of morbidity and mortality. Arch Otolaryngol Head Neck Surg 125: 149-152, 1999.

3. Ehrlich M: DNA hypomethylation in cancer cells. Epigenomics 1: 239-259, 2009.

4. Herman JG and Baylin SB: Gene silencing in cancer in association with promoter hypermethylation. N Engl J Med 349: 2042-2054, 2003.

5. Latham, KE: X chromosome imprinting and inactivation in the early mammalian embryo. Trends Genet 12: 134-138, 1996.

6. Lyko F and Brown R: DNA methyltransferase inhibitors and the development of epigenetic cancer therapies. J Natl Cancer Inst 97: 1498-1506, 2005.

7. Fang MZ, Wang Y, Ai N, Hou Z, Sun Y, Lu H, Welsh W and Yang CS: Tea polyphenol (-)-epigallocatechin-3-gallate inhibits DNA methyltransferase and reactivates methylation-silenced genes in cancer cell lines. Cancer Res 63: 7563-7570, 2003.

8. Shao C, Tan M, Bishop JA, Liu J, Bai W, Gaykalova DA, Ogawa T, Vikani AR, Agrawal Y, Li RJ, et al: Suprabasin is hypomethylated and associated with metastasis in salivary adenoid cystic carcinoma. PLoS One 7: e485582, 2012.

9. Shao C, Sun W, Tan M, Glazer CA, Bhan S, Zhong X, Fakhry C, Sharma R, Westra WH, Hoque MO, et al: Integrated, genome-wide screening for hypomethylated oncogenes in salivary gland adenoid cystic carcinoma. Clin Cancer Res 17: 4320-4330, 2011.

10. Fan X, Chen B, Xu J, Zhang H, Deng F and Xiang X: Methylation status of the PTEN gene in adenoid cystic carcinoma cells. Mol Med Rep 3: 775-779, 2010.

11. Daa T, Kashima K, Kondo Y, Yada N, Suzuki M and Yokoyama S: Aberrant methylation in promoter regions of cyclin-dependent kinase inhibitor genes in adenoid cystic carcinoma of the salivary gland. APMIS 116: 21-26, 2008.

12. Williams MD, Chakravarti N, Kies MS, Maruya S, Myers JN, Haviland JC, Weber RS, Lotan R and El-Naggar AK: Implications of methylation patterns of cancer genes in salivary gland tumors. Clin Cancer Res 12: 7353-7358, 2006.

13. Clark JC, Thomas DM, Choong PF and Dass CR: RECK - a newly discovered inhibitor of metastasis with prognostic significance in multiple forms of cancer. Cancer Metastasis Rev 26: 675-683, 2007.

14. Masui T, Doi R, Koshiba T, Fujimoto K, Tsuji S, Nakajima S, Koizumi M, Toyoda E, Tulachan S, Ito D, et al: RECK expression in pancreatic cancer: Its correlation with lower invasiveness and better prognosis. Clin Cancer Res 9: 1779-1784, 2003. 
15. Takemoto N, Tada M, Hida Y, Asano T, Cheng S, Kuramae T, Hamada J, Miyamoto M, Kondo S and Moriuchi T: Low expression of reversion-inducing cysteine-rich protein with Kazal motifs (RECK) indicates a shorter survival after resection in patients with adenocarcinoma of the lung. Lung Cancer 58: 376-383, 2007.

16. Figueira RC, Gomes LR, Neto JS, Silva FC, Silva ID and Sogayar MC: Correlation between MMPs and their inhibitors in breast cancer tumor tissue specimens and in cell lines with different metastatic potential. BMC Cancer 9: 20, 2009.

17. Zhang C, Ling Y, Xu Y, Gao L, Li R, Zhu J, Fan L and Wei L: The silencing of RECK gene is associated with promoter hypermethylation and poor survival in hepatocellular carcinoma. Int J Biol Sci 8: 451-458, 2012.

18. Zhou X, Huang S, Jiang L, Zhang S, Li W, Chen Z and Zhang D: Expression of RECK and MMP-2 in salivary adenoid cystic carcinoma: Correlation with tumor progression and patient prognosis. Oncol Lett 7: 1549-1555, 2014.

19. Kato K, Long NK, Makita H, Toida M, Yamashita T, Hatakeyama D, Hara A, Mori H and Shibata T: Effects of green tea polyphenol on methylation status of RECK gene and cancer cell invasion in oral squamous cell carcinoma cells. Br J Cancer 99: 647-654, 2008.
20. Oh J, Takahashi R, Kondo S, Mizoguchi A, Adachi E, Sasahara RM, Nishimura S, Imamura Y, Kitayama H, Alexander DB, et al: The membrane-anchored MMP inhibitor RECK is a key regulator of extracellular matrix integrity and angiogenesis. Cell 107: 789-800, 2001.

21. Echizenya M, Kondo S, Takahashi R, Oh J, Kawashima S, Kitayama H, Takahashi C and Noda M: The membrane-anchored MMP-regulator RECK is a target of myogenic regulatory factors. Oncogene 24: 5850-5857, 2005.

22. Cho CY, Wang JH, Chang HC, Chang CK and Hung WC: Epigenetic inactivation of the metastasis suppressor RECK enhances invasion of human colon cancer cells. J Cell Physiol 213: 65-69, 2007.

23. Chang HC, Cho CY and Hung WC: Downregulation of RECK by promoter methylation correlates with lymph node metastasis in non-small cell lung cancer. Cancer Sci 98: 169-173, 2007.

24. Hellebrekers DM, Griffioen AW and van Engeland M: Dual targeting of epigenetic therapy in cancer. Biochim Biophys Acta 1775: 76-91, 2007.

25. Shaw RJ, Hall GL, Lowe D, Bowers NL, Liloglou T, Field JK, Woolgar JA and Risk JM: CpG island methylation phenotype (CIMP) in oral cancer: Associated with a marked inflammatory response and less aggressive tumour biology. Oral Oncol 43: 878-886, 2007. 